\title{
Visions de la complexité. Le démon de Laplace dans tous ses états
}

\author{
Guillaume Deffuant ${ }^{1}$, Arnaud Banos ${ }^{2}$, David Chavalarias ${ }^{3}$, Cyrille Bertelle ${ }^{4}$, Nicolas Brodu ${ }^{5}$,
} Pablo Jensen ${ }^{6}$, Annick Lesne ${ }^{7}$, Jean-Pierre Müller ${ }^{8}$, Édith Perrier ${ }^{9}$, Franck Varenne ${ }^{10}$

1 Mathématiques et informatique, IRSTEA, Laboratoire d'ingénierie pour les systèmes complexes, 63178 Aubière, France

2 Géographie, CNRS, UMR 8504 Géographie-cités (CNRS/Université Paris-1 Panthéon-Sorbonne/Université Paris Diderot), 75005 Paris, France

3 Mathématiques et informatique, CNRS, UMR 8557 Centre d'analyses et de mathématiques sociales (ISC-PIF), 75013 Paris, France

4 Informatique, Université du Havre, Laboratoire d'informatique, de traitement de l'information et des systèmes (FR 3638 CNRS, ISCN), 76600 Le Havre, France

5 Informatique, INRIA Bordeaux-Sud-Ouest, GéoStat, 33405 Talence Cedex, France

6 Physique, CNRS, UMR 5672 Laboratoire de physique de l'ENS de Lyon (IXXI), 69342 Lyon Cedex 07, France

7 Biophysique, CNRS, UMR 7600 Laboratoire de physique théorique de la matière condensée (UPMC-Paris-6, Sorbonne Universités), 75252 Paris Cedex 5, France

8 Informatique, modélisation, CIRAD, UR Gestion de ressources renouvelables et environnement, 34398 Montpellier Cedex 5, France

9 Mathématiques appliquées, IRD, UMI Unité de modélisation mathématique et informatique des systèmes complexes, 93143 Bondy Cedex, France

${ }^{10}$ Épistémologie, Université de Rouen, UMR 8598 Groupe d'étude des méthodes de l'analyse sociologique de la Sorbonne, 76821 Mont-Saint-Aignan, France

Pour traiter certaines questions, que leur recherche soit finalisée ou non, des chercheurs font le choix de construire leurs objets en termes de "systèmes " ou de "problèmes complexes ». Le recours à des démarches interdisciplinaires en est souvent le corollaire, entraînant des choix épistémologiques qui doivent être assumés et explicités comme tels. C'est ainsi que l'on a pu observer l'émergence d'une «communauté du complexe » depuis une vingtaine d'années (s'appuyant, entre autres, sur la création de groupements d'intérêt scientifique constituant un réseau national). Partant du domaine des mathématiques à propos du comportement de systèmes très sensibles aux conditions initiales, la problématique s'est étendue aux questions posées par les relations entre niveaux au sein des systèmes (par exemple, de l'individu à la population), puis à la « complexité sociale ». Cet article, signé par des chercheurs dont les spécialités initiales sont très variées (des mathématiques à la géographie et la sociologie), montre bien que les « sciences du complexe » soulèvent de nombreuses questions qui appellent des pratiques interdisciplinaires. Il faudrait qu'en retour NSS s'intéresse à la façon dont les méthodes et les concepts des «sciences du complexe " retentissent dans les approches interdisciplinaires que la revue promeut. Nous invitons les lecteurs à lire, également dans ce numéro, le compte rendu des Journées de Rochebrune de 2014, organisées par cette « communauté du complexe », et qui étaient consacrées à l'interdisciplinarité.

La Rédaction

Auteur correspondant: G. Deffuant, guillaume.deffuant@irstea.fr

À propos des auteurs, É. Perrier a été directrice du Réseau national des systèmes complexes (RNSC), et G. Deffuant co-directeur. D. Chavalarias est directeur de l'ISC-PIF (Institut des systèmes complexes de Paris Île-de-France), il a succédé à A. Banos. C. Bertelle est cofondateur de 1'ISCN (Institut des systèmes complexes de Normandie). P. Jensen a été directeur de 1'IXXI (Institut Rhône-alpin des systèmes complexes). 
Mots-clés :

complexité ; systèmes complexes ; démon de Laplace

\section{Keywords:}

complexity; complex systems;

Laplace's demon
Résumé - Nous distinguons trois visions de la complexité afin de clarifier les contours de la recherche dans ce domaine. Nous utilisons le démon de Laplace comme référence pour présenter ces visions. La vision 1 brise le rêve du démon de Laplace en identifiant des systèmes particuliers qui lui résistent en mathématiques, physique et informatique. La vision 2 propose une nouvelle version du rêve de Laplace fondée sur la disponibilité récente de grandes quantités de données et de nouvelles technologies de programmation, de stockage et de calcul. La vision 3 met le démon face au défi de simuler la subjectivité et ses effets collectifs.

Abstract - Visions of complexity. New challenges for revisited Laplace's demon. We identify three different visions of complexity with the aim to clarify the research field on complex systems. We use Laplace's demon as a reference for formulating these visions. Vision 1 breaks Laplace's dream by exhibiting fundamental difficulties in mathematics, physics and computer sciences hampering to derive systemic properties from the knowledge of components characteristics and their interactions rules. Vision 2 reactivates Laplace's dream, with new demons based on increased computation power and big data availability, yet subject to the limitation identified by vision 1 . Finally, vision 3 challenges Laplace's demon to simulate subjectivity, counterfactuals, empathy and their cascading collective effects.

\section{Introduction}

Dans son sens usuel, complexité est synonyme de complication. Elle désigne une difficulté, une résistance à la compréhension ou à l'explication. Dans cette acception usuelle, la complexité dépend donc fortement du développement scientifique et technique, qui fournit de nouveaux outils ou change de perspective. Par exemple, exécuter une chaîne d'opérations incluant des racines carrées et des logarithmes était une tâche difficile, complexe, avant l'émergence de l'ordinateur. Elle ne l'est plus maintenant. Inversement, l'univers aristotélicien, dominant en Occident de l'Antiquité jusqu'à la Renaissance, se compose de quelques sphères en rotation autour de la Terre, ce qui est considérablement moins complexe que les milliards de galaxies en expansion et que la variété des corps (étoiles diverses, planètes, trous noirs, nuages cosmiques...) de l'astronomie moderne. Ainsi, dans cette acception courante, la complexité dépend des connaissances et des techniques disponibles.

Cependant, depuis une trentaine d'années, les systèmes complexes sont devenus un champ de recherche. À la suite des pionniers, le Santa Fe Institute aux États-Unis dans les années 1980 et, parallèlement en Europe, le centre de recherche animé par Prigogine à Bruxelles, des centres de recherche sur les systèmes complexes sont, par exemple, apparus en Italie, en Espagne, au Royaume-Uni, en Pologne, en Hongrie et en France. Les chercheurs ont créé une association internationale ${ }^{1}$, des revues spécialisées, et des conférences internationales se tiennent régulièrement. Le champ est particulièrement bien structuré en France avec des centres régionaux (Paris-Île-de-France, Rhône-Alpes, Rennes, Normandie) et des réseaux thématiques, l'ensemble étant intégré dans la structure d'animation et de réflexion du Réseau national des systèmes complexes

\footnotetext{
1 http://cssociety.org
}

(RNSC). Certains représentants de ce champ scientifique évoquent une science des systèmes complexes ou de la complexité (Zwirn et Weisbuch, 2010).

Que veut dire complexe pour ces chercheurs? Il ne peut évidemment s'agir uniquement du sens usuel, car alors tous les problèmes scientifiques difficiles, qui résistent aux connaissances actuelles, en feraient partie. Ce champ intégrerait alors l'ensemble de la recherche sans aucune spécificité et ne pourrait prétendre à aucune identité propre. Il est donc particulièrement important pour ce champ scientifique de s'appuyer sur une définition de la complexité correspondant à des propriétés intrinsèques du système considéré, indépendantes des circonstances et de l'avancement scientifique.

Bien sûr, de nombreux ouvrages de très grande qualité sont consacrés à cette question (Gell-Mann, 1994 ; Gribbin, 2006; Boccara, 2004 ; Miller et Page, 2007 ; Mitchell, 2009; Zwirn, 2006). Cet article se nourrit à la fois de cette littérature et de l'observation « de terrain » de la communauté de recherche dans laquelle les auteurs évoluent. Ce double ancrage nous a progressivement conduits à identifier trois grandes tendances ou trois visions de la complexité. À notre connaissance, cette catégorisation est originale, car la plupart des ouvrages tentent de présenter le domaine sous un seul visage. Nous pensons cependant qu'il est important d'identifier clairement ces points de vue différents, car les passer sous silence engendre selon nous des incompréhensions. Notre objectif dans ce texte est donc à la fois de rendre compte de cette diversité, mais aussi d'en proposer une présentation simple, afin de bien en faire apparaître les lignes de force principales.

Nous présentons tout d'abord une formulation moderne du démon de Laplace, qui nous sert de référence commune aux différentes visions de la complexité. Nous décrivons ensuite ces trois visions plus en détail. Nous discutons enfin les rapports entre ces trois visions. 


\section{Vision 1 : des systèmes qui résistent au démon de Laplace, vecteurs de nouveaux cadres conceptuels}

\section{Le démon de Laplace et sa formulation moderne}

Laplace (1814) décrit ce fameux démon de la manière suivante: "Une intelligence qui, à un instant donné, connaîtrait toutes les forces dont la nature est animée et la situation respective des êtres qui la compose embrasserait dans la même formule les mouvements des plus grands corps de l'univers et ceux du plus léger atome ; rien ne serait incertain pour elle, et l'avenir, comme le passé, serait présent à ses yeux ». Cette idée est suggérée par les succès sans précédent de la mécanique de Newton. Il nous semble intéressant de la considérer comme référence pour caractériser les systèmes complexes.

Mais nous proposons d'abord de moderniser le démon en le munissant d'un ordinateur. Supposons que le démon de Laplace observe un système défini au lieu de l'Univers entier, qu'il connaisse les positions initiales des éléments de ce système à un moment donné, et qu'il puisse écrire un programme qui traduit les dynamiques de chacun de ces éléments au cours du temps. À partir de ce programme et des conditions initiales à un instant donné, le démon peut en principe calculer n'importe quel état futur du système.

Pour que cette approche soit pertinente en pratique, il faut que le programme soit relativement court (un programme infiniment long est impossible à écrire), que son exécution soit relativement rapide pour obtenir des résultats avec une précision acceptable (de même, un temps d'exécution infiniment long fait perdre tout intérêt à l'approche ${ }^{2}$ ). Nous dirons donc qu'un système résiste au démon de Laplace et brise le rêve de Laplace si aucun programme ne satisfait ces conditions.

En fait, depuis la fin du XIX ${ }^{e}$ siècle, le rêve de Laplace a été brisé plusieurs fois. On pense, bien sûr, tout d'abord à la mécanique quantique qui rend impossible l'assertion « rien ne serait incertain pour elle ». En effet, selon cette théorie, il est physiquement impossible de connaître avec une précision parfaite à la fois la position et la vitesse d'une particule, même pour le plus intelligent des démons. Ainsi, le déterminisme qui est présupposé par Laplace est fondamentalement remis en question.

Mais certains travaux en mathématiques, informatique et en physique nous semblent avoir porté des coups tout aussi rudes en remettant en cause l'autre partie du

\footnotetext{
2 La longueur du programme minimal pour engendrer une suite d'entiers et son temps d'exécution sont utilisés pour définir précisément la complexité de cette suite d'entiers (Kolmogorov, 1963; Li et Vitanyi, 1999) et sa profondeur logique (Bennett, 1988), dont les détails ne sont pas essentiels à notre propos.
}

rêve : "l'avenir, comme le passé, serait présent à ses yeux ", même pour des systèmes déterministes relativement simples. Cette manière de briser le rêve de Laplace constitue pour nous l'identité de la vision 1 de la complexité.

\section{Complexité mathématique : sensibilité aux conditions initiales}

Poincaré, à la fin du XIXe siècle, révèle un problème majeur des systèmes newtoniens comprenant plus de deux corps : la sensibilité aux conditions initiales. Deux trajectoires du système, partant de deux points extrêmement proches, deviennent rapidement très différentes l'une de l'autre.

De telles dynamiques, mises aussi en évidence par Lorenz sur des modèles météorologiques simplifiés, ont permis de définir la notion mathématique de chaos. Ainsi, Lorenz exprimait en 1972 cette question sur la prédictibilité en météorologie : « Le battement d'ailes d'un papillon au Brésil peut-il provoquer une tornade au Texas? ». L'image est restée célèbre (parfois baptisée effet papillon) même si Lorenz lui-même dans ce papier précise qu'il faudrait considérer statistiquement la population de tous les papillons, chacun ayant autant de chance de provoquer que d'empêcher la formation d'une tempête, et donc que seules des distributions statistiques d'événements sont pertinentes. Cependant, les spécialistes s'accordent sur le fait que les non-linéarités des dynamiques météorologiques entachent les prévisions d'une incertitude irréductible, qui s'accroît rapidement avec l'horizon de prédiction.

Cette difficulté brise le rêve de Laplace, car la connaissance des conditions initiales d'un système a nécessairement une précision finie, ce qui implique que l'avenir, parfois proche pour un système très chaotique, reste nécessairement opaque au démon. Autrement dit, même si le programme du démon est très simple et rend parfaitement compte des dynamiques des éléments du système, il requiert une description de l'état initial du système qui occupe une taille mémoire infinie pour permettre de calculer l'évolution du système avec précision.

Les fractales comme les ensembles de Julia sont un autre exemple d'objets mathématiques résistant au démon de Laplace. Ces objets sont définis par une règle de calcul très simple (Mandelbrot, 1977). Cependant, tout niveau d'approximation ampute la figure d'une infinité de motifs imbriqués les uns dans les autres, qui se multiplient indéfiniment quelle que soit la petitesse de la portion d'espace considérée. Ainsi, cette approximation reste toujours infiniment mauvaise, quel que soit le temps de calcul. De tels objets, engendrés par un programme très compact mais dont le temps d'exécution tend vers l'infini, brisent également le rêve de Laplace. 


\section{Complexité de von Neumann : machine de Turing et théorème de Gödel}

La machine de Turing (Turing, 1937) constitue ellemême un défi au démon de Laplace. En effet, il est démontré qu'il n'existe aucun programme permettant de prévoir en temps fini, pour toute machine de Turing et toute entrée, si la machine s'arrête au bout d'un nombre d'opérations fini ou bien continue à fonctionner indéfiniment. Il s'agit donc d'un exemple où la connaissance complète des règles de fonctionnement d'un système (la machine de Turing universelle qui est complètement spécifiée) n'est pas suffisante pour qu'une intelligence finie (considérée comme un programme) en prédise l'ensemble des comportements possibles (pour plus $\mathrm{d}$ 'information sur ce problème qui est lié au théorème de Gödel, voir, par exemple, Delahaye, [1999]).

Von Neumann s'appuie sur cette propriété pour proposer une définition de la complexité : une machine est complexe lorsqu'il est infiniment plus facile de décrire ses éléments et leurs règles de fonctionnement que de décrire l'ensemble des comportements possibles engendrés par ces composants et leurs interactions (Dupuy, 1994).

\section{Complexité physique : systèmes complexes et émergence}

Selon la vision physicienne, un système complexe est composé de nombreux éléments en interaction, qui présentent un comportement collectif différent de celui obtenu par la simple juxtaposition des comportements individuels (voir, par exemple, Newman, 2011). Cette définition est imprégnée du cadre de la physique statistique, même si elle est étendue à des systèmes biologiques et sociaux. Ainsi, l'article classique d'Anderson (1972), intitulé "More is different », explique que le comportement collectif des éléments d'un système physique est souvent difficile (et parfois impossible) à déduire du comportement individuel de ces éléments.

Considérons, par exemple, un gaz confiné dans un compartiment. Si on lui ouvre l'accès à un autre compartiment, le gaz envahit tout l'espace disponible et la probabilité que les particules de gaz reviennent toutes en même temps dans le premier compartiment est infime. Le comportement collectif du gaz est irréversible. Cependant, le comportement de chacune des particules considérée isolément est réversible. C'est la physique statistique de Boltzmann qui permettra de comprendre ce mystère en établissant un lien statistique entre le comportement des atomes et celui du gaz. Mais pour cet exemple où ce passage entre les deux échelles a pu être établi rigoureusement, Anderson signale de nombreux exemples en physique où une telle démarche se heurte à des difficultés insurmontables.
Ce problème peut être reformulé dans le cadre de la version moderne du démon de Laplace. Supposons qu'il existe un programme qui simule les dynamiques individuelles des éléments du système. Il permet de prédire les positions de chacun de ces éléments dans le futur. Mais l'exécution de ce programme est extrêmement lourde si les éléments sont très nombreux (songeons qu'un centimètre cube d'air contient des milliards d'atomes). La question est donc de déterminer un programme plus efficace qui permette de calculer plus directement des états globaux du système (en utilisant, par exemple, des grandeurs statistiques). Ce problème de passage rigoureux entre dynamiques micro et macro est, selon Anderson, souvent très difficile, voire insoluble. La difficulté (ou l'impossibilité) de ce passage est la signature de la complexité et est souvent associée au concept d'émergence. La définition de ce concept et de ses variantes fait l'objet de nombreux débats dans lesquels nous ne pouvons entrer ici.

Les exemples d'émergence dans des modèles physiques permettent de concevoir la vie comme un processus émergeant de processus biochimiques, c'est-à-dire comme issue de ces processus tout en en étant fondamentalement différente. Nous évoquerons à nouveau l'émergence dans la vision 3 de la complexité à propos des dualités neurone/esprit et individu/société.

\section{Des cadres conceptuels nouveaux}

Dans les paragraphes précédents, beaucoup des travaux considérés mettent en évidence des limites de nos modèles ou même de la démarche scientifique. En effet, ils montrent nos limites à prédire le comportement de certains systèmes, dits complexes, même si leur dynamique se formule de façon très simple (système à trois corps, fractales, émergence ${ }^{3}$ ).

Ce rôle de briseur du rêve de Laplace est important, mais il peut aussi donner l'impression que cette recherche produit uniquement des résultats négatifs. Il nous semble important de corriger cette impression et d'insister sur des aspects plus constructifs de cette première vision de la complexité.

Tout d'abord, les travaux que nous décrivons ont changé notre regard sur le monde. Les concepts de dépendance aux conditions initiales, de changements d'échelles, de fractales ou même d'émergence ont diffusé dans de nombreux domaines scientifiques où ils ont permis des avancées.

3 La différence entre complexité et complication apparaît ici clairement. La difficulté à prédire les comportements de ces systèmes n'est pas liée à la complication de leurs mécanismes, qui peuvent être très simples. La propriété en question est par ailleurs indépendante du développement scientifique. 
Les chercheurs ont aussi défini des concepts nouveaux comme les états critiques auto-organisés, les structures dissipatives ou les rois-dragons (Sornette et Ouillon, 2012) pour caractériser des dynamiques émergentes particulières. De manière générale, la recherche s'attache justement à mieux comprendre le passage entre les dynamiques des éléments et la dynamique globale du système ou inversement (Müller, 2004 ; Lesne, 2013) et à développer des outils et des méthodes pour résoudre au moins partiellement ces difficultés.

De même, la sensibilité aux conditions initiales de nombreux systèmes dynamiques amène à développer de nouvelles méthodes permettant de faire des prédictions en probabilité sur le comportement de ces systèmes, à des échelles temporelles et spatiales limitées.

Nous avons brossé très rapidement une première vision de la complexité, issue de travaux en physique, en mathématiques et en informatique, dont la caractéristique commune est de briser le rêve de Laplace.

\section{Vision 2 : de nouveaux démons de Laplace}

Parallèlement à la reconnaissance des limites intrinsèques de nos capacités de modélisation, la croissance de la puissance de calcul des ordinateurs et des nouveaux moyens multiples de recueil et de stockage de données suscite de nouveaux espoirs, sans précédent. Il nous semble qu'une partie de la recherche sur les systèmes complexes, fondée sur ces espoirs, est porteuse d'une vision de la complexité différente de celle que nous venons de présenter.

Cette vision prend acte de la complexité au sens du rêve brisé de Laplace (émergence, sensibilité aux conditions initiales...), mais considère que, dans certains cas, il est pertinent d'appliquer le principe de base du démon - identifier tous les éléments du système et leurs conditions initiales, modéliser leur dynamique, puis simuler ces dynamiques - à des systèmes limités (et non à l'Univers entier) et pour des échelles spatiales et temporelles bien identifiées. Il s'agit donc d'utiliser la puissance informatique pour intégrer différentes facettes d'un système, correspondant souvent à des disciplines scientifiques différentes, et ainsi d'obtenir une intégration de savoirs multiples, y compris à partir de sources de données diverses.

Cette vision prend un essor particulier ces dernières années grâce à la disponibilité d'une quantité et d'une diversité de données sans précédent. Cet afflux de données, que certains ont même appelé un déluge, donne accès à des mesures multiples sur un grand nombre de phénomènes, y compris sociaux. Le XIX ${ }^{\mathrm{e}}$ siècle a déjà connu une "avalanche» de données sur la société (Hacking, 1990) qui a eu d'énormes conséquences épistémiques (naissance des branches statistiques des mathématiques et de la physique). Pour certains, cette nouvelle avalanche de données sera à l'origine d'un tournant majeur dans l'activité scientifique (Anderson, 2011).

Nous proposons tout d'abord quelques exemples illustratifs de cette vision, puis nous analysons ses traits principaux.

\section{Quelques exemples}

La modélisation de l'évolution du climat nécessite des simulations informatiques très lourdes couplant plusieurs dynamiques (atmosphère, océans, effet des nuages...), faisant appel à des compétences et des spécialités différentes et à des sources de données massives, recueillies notamment par les réseaux de capteurs divers. La simulation de tels modèles demande des puissances de calcul qui ne sont disponibles largement que depuis quelques années. La sensibilité aux conditions initiales est systématiquement évaluée pour chacun des modèles. La comparaison entre les résultats de différents modèles est également en train de se généraliser. L'élaboration de ces modèles requiert des collaborations entre informaticiens et physiciens, spécialistes de l'atmosphère et du climat.

La modélisation de la structure et de l'évolution $d^{\prime}$ Internet, avec les travaux sur les réseaux qu'elle a suscités, est un champ nouveau des sciences sociales et de la modélisation qui se développe très rapidement. Internet fournit aussi des traces accessibles de nombreuses activités, comme la recherche par exemple. Les données de publications accessibles dans de très grandes bases de données permettent de cartographier l'évolution de l'utilisation de différents termes, les collaborations, les citations (Chavalarias et Cointet, 2013 ; Grauwin et al., 2012). Ces recherches permettent aussi d'étudier la structure de grands réseaux d'interactions à partir de données et de les confronter à des travaux théoriques.

En biologie, l'un des défis actuels est de modéliser les réseaux de régulation génétique qui permettraient de prédire l'expression des gènes (voir, par exemple, Davidson et Levin, 2005). Les recherches dans ce domaine mobilisent diverses approches de modélisation prenant en compte les sources de données massives issues des techniques de génomique (séquençage de nouvelle génération, transcriptomique pour mesurer le niveau d'expression des gènes, immunoprécipitation permettant de déterminer les facteurs liés in vivo à l'ADN...) et d'épigénomique (profils de marques épigénétiques le long du génome). Cette même problématique se retrouve dans la modélisation multiéchelles des organes (cœur, reins...) ou du système immunitaire entier, par exemple. Jusqu'à une date récente, le système immunitaire était en effet modélisé dans certains de ses 
aspects seulement à l'aide de formalismes distincts (Ratzé et al., 2007), alors qu'on observe aujourd'hui le recours à des simulations intégratives multiéchelles à base de métamodélisation informatique (Thomas-Vaslin et al., 2013). Les modèles de simulation de la structure des plantes font aussi appel à plusieurs formalismes différents correspondant à différentes dynamiques ou propriétés. Ainsi, pour formaliser le développement architectural d'un arbre, il faut, par exemple, distinguer des probabilités de ramification, le graphe d'ordonnancement des branches, la longueur des axes et leur distance mutuelle, les lois physiques de flexion des branches. Chacun de ces aspects correspond à un sousmodèle qui fait appel à un formalisme particulier. Les sous-modèles sont couplés entre eux par des transmissions de valeurs de variables, mais aucun formalisme unifiant n'est disponible (Varenne, 2008).

Le projet «Global Epidemic Model » a pour objectif de prédire la propagation des épidémies (Van den Broeck et al., 2011). Le modèle prend en compte la population mondiale et ses différents types de déplacement en s'appuyant sur de nombreuses sources de données. En particulier, les zones où les données sont abondantes sont utilisées pour définir des corrélations qui permettent de combler les données manquantes ailleurs. Ensuite, un modèle de propagation de l'épidémie détermine les contaminations dans le temps à partir des foyers initiaux, des déplacements et des contacts des agents initialement contaminés. Ce modèle a notamment été testé avec succès sur le virus de la grippe H1N1 (Tizzoni et al., 2012). Il vise à aider les responsables politiques à prendre des mesures de prévention.

Ces exemples présentent une grande diversité, mais ils dépendent tous de la puissance et de la capacité de traitement des données des ordinateurs (et souvent de réseaux d'ordinateurs). Ils s'appuient aussi sur des évolutions technologiques qui facilitent l'utilisation de grandes quantités de données et la programmation de modèles fondés sur l'interaction de composants éventuellement hétérogènes.

\section{Le rôle central de mutations technologiques profondes}

Pour pouvoir utiliser les quantités de données disponibles dans des projets de modélisation, il est souvent nécessaire au préalable d'étudier ces données afin d'en faire ressortir des régularités, des structures, qui pourront ensuite être comparées à celles obtenues à partir des modèles. Ainsi, les travaux de la vision 2 utilisent :

- les méthodes et outils de visualisation et d'exploration de grandes masses de données. Ces outils sont en croissance rapide, avec le développement d'entrepôts de données et d'outils de visualisation spécifiques. On peut noter des développements particuliers pour visualiser des réseaux d'interactions sur le web (voir, par exemple, Chavalarias et Cointet, 2008) ou la visualisation de dynamiques d'embryogénèse permettant d'identifier les trajectoires individuelles des cellules ou encore de structuration de biofilms bactériens. D'autres outils de visualisation de données s'inspirent de la sociologie de l'acteur-réseau (Latour, 2006 ; Latour et al., 2013) ; - les méthodes et outils d'apprentissage statistique et de fouilles de données. Ces méthodes cherchent à établir automatiquement des régularités statistiquement robustes (motifs, patterns en anglais) dans les données. Pour Bourgine (2008), la détection des régularités essentielles et leur représentation pertinente sont un premier niveau de modélisation très important, appelé « reconstruction phénoménologique ».

Ces problèmes de visualisation et d'apprentissage sont l'objet de recherches très actives, et connexes au domaine des systèmes complexes.

La deuxième évolution importante concerne le développement de cadres informatiques comme la DEVS (Discrete Event System Specification) (Zeigler, 1987) ou des environnements de programmation à base d'agents qui facilitent la conception et la mise en œuvre des modèles de la vision 2 de la complexité. En effet, ils facilitent la programmation des ensembles de composants dynamiques couplés, éventuellement hétérogènes. En particulier, les langages informatiques récents (à objets ou à agents) permettent de représenter des objets par des attributs, et des comportements dynamiques qui les caractérisent.

Ces évolutions changent certaines pratiques habituelles de modélisation. En effet, ces programmes se différencient des modèles mathématiques ou physiques plus classiques. Ils pourraient être décrits de manière exhaustive formellement ou mathématiquement, mais une telle description est en général inutilisable. Certains protocoles généraux tentent de normaliser la présentation de ces modèles dans les articles scientifiques (Grimm et al., 2005).

Comme dans le cas des modèles théoriques que nous avons répertoriés dans la vision 1 de la complexité, même si les différentes parties de ces modèles sont parfaitement connues, leur composition peut engendrer des effets difficiles à prévoir a priori, qu'il faut explorer et comprendre. Cela implique de nouvelles pratiques pour explorer et tester systématiquement ces modèles, mais aussi souvent pour modéliser leurs comportements intégrés, statistiquement ou mathématiquement. Certains parlent alors de "re-mathématisation » du modèle (Varenne, 2008). Ce sont des outils de la vision 1 qui doivent être adaptés aux modèles de la vision 2, en particulier à l'hétérogénéité des formalismes et des masses de données. 


\section{Une identité en devenir ?}

Cette vision de la recherche sur les systèmes complexes, adoptant par défaut la stratégie du démon de Laplace, vise à construire des connaissances nouvelles en intégrant des savoirs disciplinaires et des données dans des modèles nouveaux. Elle mobilise souvent des compétences disciplinaires spécifiques pour différentes parties du modèle, en plus des compétences des informaticiens. Elle suppose des données ou des connaissances disponibles sur les différentes parties du modèle qui se situent souvent à des échelles de temps et d'espace différentes (comme dans les modèles d'épidémiologie ou du climat). Ses résultats ou ses réalisations peuvent être revendiqués par les différentes disciplines participantes et laisser dans l'ombre ce qui est spécifique à la recherche sur la complexité, d'autant que les objets qu'elle modélise entrent parfois dans le champ d'une discipline (par exemple, la biologie pour le système immunitaire).

Par ailleurs, dans cette vision, la complexité est liée à la quantité de données, à la puissance de calcul déployée et à l'hétérogénéité des objets et des disciplines mobilisées. Il est délicat de préciser, sur ces quantités, un seuil qui distinguerait le complexe du non-complexe, selon cette vision dont l'identité est donc moins tranchée pour $l^{\prime}$ instant que celle de la vision 1 .

Cependant, les dispositifs intégrateurs nouveaux de la vision 2 sont portés par les progrès des technologies de l'information, et l'amplification de leur développement fait peu de doutes. Ils produisent des connaissances nouvelles par les possibilités accrues d'intégration qu'ils offrent mais créent également souvent des zones d'ignorance dans la mesure où ils échappent en général à la compréhension d'un seul individu, soit à cause de la quantité et de la diversité de leurs objets, soit à cause de la diversité des disciplines qu'ils mobilisent. La vision 2 peut ainsi être à l'origine d'avancées scientifiques majeures et de bouleversements dans notre utilisation des modèles qui pourraient affirmer son identité à l'avenir.

\section{Vision 3 : complexités du sujet connaissant, social}

Nous considérons maintenant un démon de Laplace cherchant à simuler des agents dotés d'une subjectivité. Pour les réductionnistes, le démon doit se référer aux états neuronaux ou physicochimiques du système. Mais pour les non-réductionnistes, il est légitime et plus efficace d'essayer de reconstituer ce que perçoivent et ressentent les agents pour prédire leur comportement. Le démon de Laplace doit donc selon eux « simuler » la subjectivité de chacun des agents, pour en simuler le comportement. Nous évoquons les arguments qui nous paraissent justifier une troisième vision de la complexité, inspirée par ce problème.

\section{Perceptions, anticipations et décisions d'actions}

Considérons donc des animaux relativement proches de nous, des mammifères, par exemple. Immédiatement, nous avons tendance à interpréter leurs comportements comme liés à un point de vue individuel sur le monde comprenant des émotions, des désirs, des craintes, des espoirs. Nagel (1974) utilisait l'expression «l'effet que cela fait d'être » un autre être vivant, comme une chauvesouris, par exemple («what is it like to be a bat»). Cette perception du monde, ce vécu d'un point de vue particulier, peuvent être considérés comme un effet émergent au sens de la vision 1 . Il s'agirait d'un agencement spécifique des activités neuronales, de même que la supraconductivité est une propriété émergente d'une configuration particulière des particules (Searle, 1995).

Cependant, on peut mesurer la supraconductivité par des expériences répétables sur lesquelles les physiciens peuvent parvenir à un accord solide. Mais comment faire la même chose pour des perceptions auxquelles par définition seul le système considéré a accès ? Comment même être certain de leur existence ? Il n'existe pas de mesure objective de l'état ressenti d'un système cognitif. De plus, certains systèmes peuvent avoir une perception totalement différente de la nôtre, à laquelle il nous est pratiquement impossible d'accéder, même par imagination (Nagel, 1974). Donc le phénomène émergent considéré présente une différence fondamentale avec les propriétés émergentes habituellement considérées dans la vision 1 . Cette difficulté supplémentaire est pour nous une spécificité importante de la vision 3.

Cette spécificité est étroitement liée à un vieux problème philosophique : celui des relations entre le corps et l'esprit. Ce problème alimente le débat sur la causalité descendante. Est-il possible que des propriétés émergentes engendrent de nouvelles lois causales qui contraignent les éléments dont elles émergent ? Dans ce cas précis, cela se traduit par: les états mentaux émergents, ressentis par le sujet, tels que le désir, la volonté, la crainte, sont-ils la cause des actions du sujet ? Autrement dit, l'esprit peut-il agir sur la matière ? À nouveau, il nous semble que cette question prend une signification particulière par rapport à celle de la causalité descendante en général, car nous ressentons tous une capacité à prendre des décisions qui sont la cause de nos actions.

Ce dualisme ressenti est généralement considéré par les chercheurs actuels comme une illusion. Cependant, il est aussi généralement admis que ces descriptions ont un pouvoir heuristique qui facilite la compréhension et l'explication. L'idée de "simplexité " (Berthoz, 2009) se nourrit en partie de ce contraste entre la description scientifique complète des processus 
physiques extérieurs auxquels le sujet est confronté, et la perception interne de la situation par le système, qui se fonde souvent sur quelques points saillants associés à des émotions particulières.

Par exemple, il est fréquent de prédire l'action d'un agent à partir des motivations qu'on lui prête et de l'hypothèse qu'il agira rationnellement pour les satisfaire, en anticipant les conséquences de ses actions. Cette démarche fait apparaître une difficulté nouvelle : le traitement de situations contrefactuelles. En effet, les agents considèrent des situations potentielles, contrefactuelles, qui dépendent de leurs actions (ou des actions des autres). Le démon de Laplace doit donc simuler ces mondes contrefactuels pour chacun des agents. Certains de ces problèmes sont traités en théorie des jeux ou en théorie du contrôle ou de la viabilité (Aubin, 1991) dans des situations simplifiées.

\section{Complexité et opacité de la subjectivité}

La combinatoire de l'exploration des mondes contrefactuels ne doit pas éclipser les difficultés plus fondamentales d'attribution d'états et de processus mentaux. Le ressenti d'un sujet peut considérablement évoluer en fonction des circonstances ou de l'attention qu'il lui prête. Certaines traditions philosophiques (phénoménologie) ou spirituelles (bouddhisme) travaillent justement à explorer, maîtriser et faire évoluer ces perceptions. Les individus entraînés à ces pratiques ont des perceptions particulières (attestées par des activations neuronales spécifiques).

Par ailleurs, les recherches récentes liées à la neurobiologie attribuent plusieurs niveaux à la conscience. Par exemple, Edelman (1990) distingue la conscience d'épisodes temporels isolés les uns des autres, qu'il appelle présent remémoré, et la conscience sociale de soi dans le temps, qu'il appelle conscience d'ordre supérieure. Damasio (1995) propose des niveaux un peu différents qui sont surtout expliqués par des mécanismes neuronaux très différents. Ces niveaux de conscience sont attestés notamment par l'observation de différentes pathologies. Ils donnent donc l'image d'une conscience multiple dans laquelle une collaboration, mais aussi des conflits peuvent émerger entre les niveaux. Ces travaux font écho à la pluralité du soi défendue par la psychanalyse. Ainsi donc, la simplicité du monde perçu par les agents pourrait n'être qu'une reconstruction a posteriori qui en gomme les contradictions et les incertitudes. La tâche du démon de Laplace pour simuler ces états internes se heurte à ces difficultés.

De plus, même si le démon de Laplace a un accès direct au ressenti des agents qu'il doit simuler, cela ne lui donne pas forcément accès à des prédictions fiables de leur comportement. En effet, de nombreux travaux montrent que certains aspects de notre comportement nous sont opaques. Nous ne comprenons pas toujours nos actes et nous percevons parfois des choses à notre insu. De telles situations sont particulièrement mises en évidence dans des cas pathologiques comme ceux des cerveaux divisés (Gazzaniga, 2005) ou de la vision aveugle (Celesia, 2010). L'expérience quotidienne de chacun et la littérature nous en fournissent de nombreux exemples.

Ainsi donc, si le démon de Laplace doit simuler le ressenti des agents pour fournir une compréhension véritable de leur comportement, il est clair que cette tâche se heurte à des difficultés très spécifiques : multiplicité et instabilité du soi et de la conscience, opacité à soi-même de certaines de nos actions.

En fait, il semble que nous soyons équipés de dispositifs spécifiques pour réaliser en partie cette tâche de simulation des états internes d'autrui, grâce à nos neurones miroirs (Rizzolatti et al., 2004). Même si leur existence chez l'homme est mise en doute par certains chercheurs, la découverte de ces neurones miroirs chez les chimpanzés a suscité un grand nombre de recherches et de réflexions sur les bases neuronales de notre capacité d'empathie, c'est-à-dire notre capacité à se mettre à la place des autres (Gallese et Goldman, 1998 ; Decety et Jackson, 2004). Mais cette capacité complique encore la tâche du démon de Laplace, car il doit simuler des agents qui se simulent les uns les autres.

\section{Complexité sociale}

Tournons-nous maintenant vers les dynamiques sociales: comportements collectifs, création d'institutions, partage de valeurs, etc. Il nous paraît tout d'abord important de souligner que notre vision de ces dynamiques est depuis longtemps marquée par les concepts de la complexité au sens de la vision 1 . Par exemple, la «main invisible » d'Adam Smith (1776), fondatrice en économie, peut être vue comme une émergence. En effet, dans ce schéma, les égoïsmes individuels sont censés se combiner pour procurer finalement un plus grand bienêtre collectif que dans une société où les individus sont altruistes.

Cependant, les travaux plus récents sur la complexité mentionnés dans la vision 1 ont fait évoluer fortement ces premières conceptions des dynamiques sociales considérées chez Hegel ou chez Marx comme des lois inéluctables ${ }^{4}$. Ces travaux permettent en effet de concevoir une dynamique sociale à la fois soumise à des tendances émergentes fortement structurantes, mais aussi sujette à des bifurcations imprévisibles, potentiellement issues d'événements très locaux, qui prennent de l'importance par des effets de cascades. Cette contribution de la vision 1 à notre perception de

\footnotetext{
4 A. Renaut (1989) défend l'idée que ces conceptions de dynamiques sociales collectives émergentes sont toutes inspirées par la monadologie de Leibniz.
} 
la société, donnant crédit à la société ouverte de Popper (1945), est donc majeure.

De plus, comme nous l'avons suggéré dans les paragraphes précédents, les dynamiques d'agents dotés de subjectivité sont particulières et engendrent des dynamiques collectives particulières. L'homme social est par nature incomplet, il imite (Tarde, 1898) et se nourrit d'autrui (Todorov, 1998). René Girard (1972) propose le mimétisme du désir comme une dynamique fondamentale permettant d'expliquer cette caractéristique. Cette réflexion rejoint la Théorie des sentiments moraux d'A. Smith, pour lequel se mettre (partiellement) à la place de l'autre est la dynamique fondamentale des relations sociales. Plus particulièrement, Smith considère que notre capacité à se mettre à la place de l'autre qui se met à la place de soi, est le fondement de la conscience d'être social humain (Dupuy, 1992, 1994). Il y aurait donc ici une forme de complexité nouvelle engendrée par ces interactions particulières inconnues des systèmes biologiques ou physiques.

Cette imbrication des empathies joue aussi un rôle fondamental dans la création d'objets sociaux comme les langues naturelles, les religions (Deffuant, 1998) ou la monnaie (Aglietta et Orléan, 2002). Ces objets émergent de dynamiques subjectives imbriquées et présentent donc une forme de complexité différente de celle des objets considérés dans la vision 1 . En général, ces thèses d'une complexité particulière de l'homme et de la société sont assez peu formalisées. Mais il faut signaler quand même certaines tentatives s'appuyant sur la théorie des jeux (Eber, 2004 ; Chavalarias, 2006) ou la théorie des conventions (Boltanski et Thévenot, 1991).

Ainsi, cette vision amène à une grande modestie sur notre capacité de compréhension et de prévision des évolutions sociales. En particulier, selon la vision 3, un point de vue unique sur une situation sociale est en général partiel et mutilant. Cette vision peut donc se retrouver dans des recherches sur des outils et méthodes pour prendre en compte les points de vue d'acteurs divers :

- la modélisation participative, qui vise à prendre en compte le point de vue des personnes concernées par le modèle (porteurs d'enjeux) et les points de vue d'experts de différentes disciplines. La démarche Commod développée notamment au Cirad constitue un exemple de cette tendance (Étienne, 2010). Cette démarche est particulièrement importante lorsque les modèles visent à être utilisés par un certain public: il est alors essentiel de prendre en compte le point de vue des futurs utilisateurs ;

- le développement d'outils informatiques permettant d'exprimer et de faire jouer entre eux une multiplicité de points de vue. Ces outils pourraient à l'avenir généraliser des pratiques de consultations itérées sur différents problèmes, visant éventuellement des populations importantes concernées par un même problème (territoires, entreprises...) (Müller et Aubert, 2013).

Ainsi, selon cette vision, les questions posées par la complexité rejoignent des débats philosophiques anciens. Les concepts développés dans la vision 1 contribuent fortement à ce débat, mais ils semblent aussi insuffisants.

\section{Discussion}

Dans cette dernière partie, nous comparons les visions entre elles et évoquons leurs articulations possibles. Puis nous revenons sur ce qui fait pour nous leur utilité.

La vision 1 a l'identité scientifique la plus claire et les contributions les plus abouties. De plus, ces contributions ont un impact très significatif sur des débats de fond concernant les sciences en général :

- la sensibilité aux conditions initiales donne un substrat scientifique à l'expérience courante selon laquelle de petites causes peuvent produire de grands effets. Elle trace également des limites à notre capacité à prévoir précisément certains phénomènes qui garderont toujours une incertitude intrinsèque ;

- la notion d'émergence donne des arguments en faveur d'une position non réductionniste. Il est vain de chercher à expliquer tous les phénomènes à partir des lois de la physique des particules. Les lois établies à d'autres échelles sur d'autres phénomènes ont la même légitimité.

Les visions 2 et 3 sont moins bien formalisées et leurs contributions n'ont pas le même retentissement. Cependant, même si elles utilisent les résultats des travaux de la vision 1, les visions 2 et 3 développent des directions de recherche originales qu'il nous semble important de distinguer. Alors que la vision 1 est fortement influencée par les mathématiques et la physique, la vision 2 l'est par l'informatique et la vision 3 par les sciences humaines et sociales. Les défis qui structurent les trois visions nous semblent fondamentalement différents les uns des autres.

La vision 2 est en cours de structuration et son importance va sans doute s'accroître. En effet, les dispositifs informatiques en réseau de recueil et de stockage de données, couplés à des systèmes de calcul vont sans doute se multiplier dans les années qui viennent (notamment avec les dispositifs informatiques en "nuage »). Se développeront probablement de multiples démons de Laplace, limités et imparfaits, que nous pourrons interroger, comme nous le faisons avec les moteurs de recherche sur Internet actuellement. Peut-être ces démons nous observeront-ils aussi constamment, par les réseaux de caméras de surveillance, les satellites, nos opérations bancaires, nos communications téléphoniques... On le voit, les enjeux scientifiques et techniques 
rejoignent rapidement ici la vie concrète de la cité et son éthique.

Par ailleurs, soulignons que les visions 1 et 2 ont des connexions entre elles. Pour comprendre certains aspects de modèles de la vision 2, les chercheurs recourent alors aux approches de la vision 1, qui sont plus familières aux physiciens ou aux mathématiciens.

La vision 3 pour sa part se situe au cœur de débats scientifiques et philosophiques majeurs: quel statut scientifique pour la subjectivité ? Quelle démarche scientifique pour l'incorporer à nos modèles ? Il s'agit en particulier de déterminer la spécificité de la complexité humaine et celle du sujet humain dans ses dimensions cognitives et sociales. Le concept d'émergence de la vision 1, point de départ intéressant, est sujet d'âpres débats entre chercheurs (scientifiques ou philosophes), notamment à propos de la causalité descendante. Les méthodes développées par la vision 2 peuvent aussi contribuer à l'avancement des questions posées par la vision 3. On le voit, par exemple, avec les nouveaux dispositifs expérimentaux en réseau (Thurner et al., 2012) qui permettent de recueillir des traces d'actions et de perceptions de dizaines de milliers de sujets. Cependant, il nous semble que les questions posées par la vision 3 sont fondamentalement différentes de celles posées par les deux premières visions.

En effet, pour certains chercheurs en sciences humaines, la nature même de la complexité humaine la rend par essence incompatible avec des formalisations mathématiques ou même logiques. La complexité serait donc plus radicale que celle observée dans les systèmes complexes de la vision 1, qui peuvent se décrire mathématiquement (ou informatiquement), même si cette description a certaines limites. Pour d'autres chercheurs, au contraire, la complexité humaine exigerait plutôt des modélisations nouvelles, différentes de celle des objets physiques, exprimant, par exemple, des imbrications de points de vue. Ces débats sont encore très ouverts et vifs dans la communauté des sciences humaines et sociales et il est probable qu'ils le resteront encore longtemps, tant les points de vue sont difficiles à concilier. Notre ambition n'est donc certainement pas de trancher ces controverses. Plus modestement, le paysage que nous avons dessiné vise à clarifier les débats sur les systèmes complexes et la complexité. En effet, ces derniers sont souvent confus lorsque les protagonistes se réfèrent à des visions différentes sans que cela soit explicite. Nous nourrissons donc l'espoir que ce texte, fruit d'une longue collaboration interdisciplinaire entre de nombreux acteurs de la recherche, fera progresser ces débats.

\section{Remerciements}

Des versions préliminaires de ce texte ont été discutées de manière approfondie au comité de pilotage du Réseau national des sciences de la complexité (RNSC) et lors des rencontres du RNSC à Roscoff en mai 2011.

\section{Références}

Aglietta, M., Orléan, A., 2002. La monnaie entre violence et confiance, Paris, Odile Jacob.

Anderson, P.W., 1972. More is different, Nature, 177, 393-396.

Anderson, C., 2011. The end of theory. The data deluge makes the scientific method obsolete, Wired Magazine, http:// www.wired.com/science/discoveries/magazine/16-07/ pb_theory.

Aubin, J.-P., 1991. Viability theory, Boston, Birkhauser.

Bennett, C.H., 1988. Logical depth and physical complexity, in Herken, R. (Ed.), The universal Turing machine. A half-century survey, Oxford, Oxford University Press, 227-257.

Berthoz, A., 2009. La simplexité, Paris, Odile Jacob.

Boccara, N., 2004. Modeling complex systems, New York, Springer.

Boltanski, L., Thévenot, L., 1991. De la justification. Les économies de la grandeur, Paris, Gallimard.

Bourgine, P., 2008. Les systèmes complexes obéissent-ils à des lois?, in Bourgine, P., Chavalarias, D., Cohen-Boulakia, C., Déterminismes et complexités : du physique à l'éthique. Autour d'Henri Atlan, Paris, La Découverte.

Celesia, G.G., 2010. Visual perception and awareness. A modular system, Journal of Psychophysiology, 24, 2, 62-67.

Chavalarias, D., 2006. Metamimetic games. Modeling metadynamics in social cognition, Journal of artificial societies and social simulations, 9, 2, http:/ /jasss.soc.surrey.ac.uk/9/2/5.html.

Chavalarias, D., Cointet, J.-P., 2008. Bottom up scientific field detection for dynamical and hierarchical science mapping. Methodology and case study, Scientometrics, 75, 1, 37-50, https:/ /hal.inria.fr/file/index/docid/126092/filename/ ScientometricsV1.pdf.

Chavalarias, D., Cointet, J.-P., 2013. Phylomemetic patterns in science evolution. The rise and fall of scientific fields, PLoS ONE, 8, 2, e54847, doi:10.1371/journal.pone.0054847.

Damasio, A., 1995. L'erreur de Descartes. La raison des émotions, Paris, Odile Jacob.

Davidson, E., Levin, M. (Eds), 2005. Gene regulatory networks, Proceedings of the national academy of sciences of the United States of America, special issue, 102, 14.

Decety, J., Jackson, P.L., 2004. The functional architecture of human empathy, Behavioral and cognitive neuroscience reviews, 3, 71-100.

Deffuant, G., 1998. Les modèles cognitifs à l'épreuve des formes du religieux. Proposition de directions de recherche centrées sur l'empathie, Intellectica , 26/27, 89-109. 
Delahaye, J.-P.,1999. Information, complexité et hasard, Paris, Hermès Science.

Dupuy, J.-P., 1992. Introduction aux sciences sociales, Paris, Ellipses.

Dupuy, J.-P., 1994. Aux origines des sciences cognitives, Paris, La Découverte.

Eber, N., 2004. Théorie des jeux, Paris, Dunod.

Edelman, G.M., 1990. The remembered present. A biological theory of consciousness, New York, Basic Books.

Étienne, M. (Ed.), 2010. La modélisation d'accompagnement. Une démarche participative en appui au développement durable, Versailles, Quæ.

Gazzaniga, M.S., 2005. Forty-five years of split-brain research and still going strong [Review], Nature Reviews Neuroscience, 6, 653-659.

Gallese, V., Goldman, A., 1998. Mirror neurons and the simulation theory of mindreading, Trends in cognitive sciences, 2, 12, 493-501.

Girard, R., 1972. La violence et le sacré, Paris, Grasset.

Gell-Mann, M., 1994. Le quark et le jaguar. Voyage au cœur du simple et $d u$ complexe, Paris, Flammarion.

Grauwin, S., Beslon, G., Fleury, E., Franceschelli, S., Robardet, C., Rouquier, J.B., Jensen, P. 2012. Complex systems science: dreams of universality, interdisciplinarity reality, Journal of the American Society for Information Science and Technology, 63, 7, 1327-1338.

Gribbin, J., 2006. Le chaos, la complexité et l'émergence de la vie, Paris, Flammarion.

Grimm, V., Revilla, E., Berger, U., Jeltsch, F., Mooij, W.M, Railsback, S.F., Thulke, H.-H., Weiner, J., Wiegand, T., DeAngelis, D.L., 2005. Pattern-oriented modeling of agentbased complex systems. Lessons from ecology, Science, 310, 987-991.

Hacking, I., 1990. The taming of chance, Cambridge, Cambridge University Press.

Kolmogorov, A., 1963. On tables of random numbers, Sankhyā, Series A, 25, 369-375.

Laplace, P.S., 1814. Essai philosophique sur les probabilités, Paris, Courcier.

Leibniz, G.W., 1991 [1 $1^{\text {re }}$ éd.: 1714]. La Monadologie, Paris, Librairie générale française.

Li, M., Vitanyi, P., 1997 [2nd ed.]. An introduction to Kolmogorov complexity and its applications, Berlin, Springer.

Lingnau, A., Gesierich, B., Caramazza, A., 2009. Asymmetric fMRI adaptation reveals no evidence for mirror neurons in humans, Proceedings of the national academy of sciences of the Unites States of America, 106, 24, 9925-9930, doi: 10.1073/ pnas.0902262106.

Latour, B., 2006. Changer de société, refaire de la sociologie, Paris, La Découverte.

Latour, B., Jensen, P., Venturini, T., Grauwin, S., Boullier, D., 2013. Le tout est toujours plus petit que ses parties, Réseaux, $177,1,197-232$.

Lesne, A., 2013. Multiscale analysis of biological systems, Acta Biotheoretica, 61, 1, 3-19.

Lorenz, E.N., 1963. Deterministic non-periodic flow, Science, 20, 130-141.

Lorenz, E.N., 1972. Predictability. Does the flap of a butterfly's wings in Brazil set off a tornado in Texas? Communication au $139^{\text {th }}$ meeting of the American Association for the
Advancement of Science, Boston, December 29, http:// eaps4.mit.edu/research/Lorenz/Butterfly_1972.pdf (communication publiée dans Lorenz, E.N., 1993. Predictability. Does the flap of a butterfly's wings in Brazil set off a tornado in Texas? in Lorenz, E.N., The essence of chaos, Seattle, University of Washington Press).

Mandelbrot, B.B., 1982. The fractal geometry of nature, San Francisco, W.H. Freeman.

Miller, J.H., Page, S.E., 2007. Complex adaptive systems. An introduction to computational models of social life, Princeton (New Jersey), Princeton University Press.

Mitchell, M., 2009. Complexity. A guided tour, New York, Oxford University Press.

Müller, J.-P., 2004. Emergence of collective behaviour and problem solving, in Omicini, A., Petta, P., Pitt, J. (Eds), Engineering societies in the agents world IV. 4th International Workshop, ESAW 2003, London, October 29-31, Berlin, New York, Springer, 1-21.

Müller, J.-P., Aubert, S., 2013. Incorporating institutions, norms and territories in a generic model to simulate the management of renewable resources, Artificial Intelligence and Law, 21, 1, 47-78, doi: 10.1007/s10506-012-9133-8.

Nagel, T., 1974. What is it like to be a bat?, Philosophical Review, 83, 4, 435-50.

Newman, M.E.J., 2011. Complex systems. A survey, http:// arxiv.org/pdf/1112.1440.pdf.

Popper, K., 1945. The open society and its enemies, London, Routledge.

Ratzé, C., Gillet, F., Müller, J.-P., Stoffel, K., 2007. Simulation modelling of ecological hierarchies in constructive dynamical systems, Ecological complexity, 4, 1/2, 13-25.

Renaut, A., 1989. L'ère de l'individu, Paris, Gallimard.

Rizzolatti, G., Craighero, L., 2004. The mirror-neuron system, Annual review of neuroscience, 27, 169-192.

Searle, J., 1995. La redécouverte de l'esprit, Paris, Gallimard.

Smith, A., 1798. Théorie des sentiments moraux, Paris, F. Buisson.

Smith, A. 1880 [1 ${ }^{\text {re }}$ éd. : 1776]. Recherches sur la nature et les causes de la richesse des nations, Paris, Guillaumin.

Sornette, D., Ouillon, G., 2012. Dragon-kings. Mechanisms, statistical methods and empirical evidence, European Physical Journal Special Topics, 205, 1-26.

Tarde, G., 1898. Les lois sociales. Esquisse d'une sociologie, Paris, F. Alcan.

Thomas-Vaslin, V., Six, A., Ganascia, J.G., Bersini, H., 2013. Dynamical and mechanistic reconstructive approaches of T lymphocyte dynamics. Using visual modelling languages to bridge the gap between immunologists, theoreticians and programmers, Frontiers in immunology, 4, 300, 1-6.

Thurner, S., Szell, M., Sinatra, R., 2012. Emergence of good conduct, scaling and zipf laws in human behavioral sequences in an online world, PLoS ONE, 7, 1, e29796, doi:10.1371/journal.pone.0029796.

Tizzoni, M., Bajardi, P., Poletto, C., Ramasco, J., Balcan, D., 2012. Real-time numerical forecast of global epidemic spreading: case study of 2009 A/H1N1pdm, BMC Medicine, 10, 165.

Todorov, T., 1998. Le jardin imparfait. La pensée humaniste en France, Paris, Grasset.

Turing, A.M., 1937. On computable numbers, with an application to the Entscheidungsproblem, Proceedings of the London Mathematical Society, 2, 42, 230-265. 
Van den Broeck, W., Gioannini, C., Gonçalves, B., Quaggiotto, M., Colizza, V., Vespignani, A., 2011. The GLEaMviz computational tool, a publicly available software to explore realistic epidemic spreading scenarios at the global scale, BMC Infectious Diseases, 11, 37.

Varenne, F., 2008. Modèles et simulations. Pluriformaliser, simuler, remathématiser, Matière Première, 3, 153-180.
Zeigler, B., 1987. Hierarchical, modular discrete-event modelling in an object-oriented environment, Simulation, 49, 5, 219-230.

Zwirn, H., 2006. Les systèmes complexes. Mathématiques et biologie, Paris, Odile Jacob.

Zwirn, H., Weisbuch, G., 2010. Qu'appelle-t-on aujourd'hui sciences de la complexité ?, Paris, Vuibert.

Reçu le 3 mars 2013. Accepté le 25 septembre 2014. 